\title{
UNIQUENESS IN ELASTODYNAMICS OF COSSERAT AND MICROPOLAR MEDIA*
}

\author{
Bx M. SHAHINPOOR and G. AHMADI (Pahlavi University Shiraz, Iran)
}

1. Introduction. The uniqueness theorems for classical elastostatics were first established by Kirchhoff [1]. The counterpart uniqueness theorem for classical elastodynamics were first given by Neumann [2]. Both works [1], [2] rested on the assumption that the elastic constants satisfy inequalities which are necessary and sufficient for the positive-definiteness of the strain energy function. The theorems of [1], [2] thus presupposed that

$$
\mu>0, \quad-1<\sigma<\frac{1}{2},
$$

where $\mu$ and $\sigma$ are, respectively, shear modulus and Poisson's ratio of the material.

Gurtin and Sternberg [3] have shown that with the limitation to the first boundaryvalue problem of classical elastodynamics (surface displacements prescribed) and for bounded domains, the inequalities (1) may be relaxed without loss of uniqueness. Thus they obtained

$$
\mu \geq 0, \quad-\infty<\sigma<\frac{1}{2}, \quad 1 \leq \sigma<\infty,
$$

or, equivalently,

$$
c_{1}^{2}=\mu / \rho \geq 0, \quad c_{2}^{2}=2(1-\sigma) \mu /(1-2 \sigma) \rho \geq 0,
$$

where $c_{1}$ and $c_{2}$ are the distortion and dilational wave velocities in a medium of unlimited extent, respectively.

As far as the elastodynamics of Cosserat and micropolar media are concerned, certain inequalities have been proposed by Mindlin and Tiersten [4] for Cosserat media ${ }^{1}$ and by Eringen [5] for micropolar media which ensure the uniqueness of solutions for the displacements field and microdeformations. Both uniqueness theorems in [4] and [5] again rest on the assumption of positive-definiteness of the strain energy density. The theorems in [4] for a Cosserat media presuppose that

$$
\mu>0, \quad 3 \lambda+2 \mu>0, \quad\left|\eta^{\prime}\right|<\eta, \quad \eta>0,
$$

where $\mu, \lambda, \eta^{\prime}, \eta$ are all material constants such that the linear constitutive equations for the material are

$$
\begin{aligned}
\tau^{*} & =\lambda \nabla \cdot \mathbf{u I}+\mu(\nabla \mathbf{u}+\mathbf{u} \nabla), \\
\mathfrak{u}^{d} & =2 \eta \nabla \nabla \times \mathbf{u}+2 \eta^{\prime} \nabla \times \mathfrak{u} \nabla,
\end{aligned}
$$

in which $\mathbf{u}$ is the deformation field, $\tau$ and $\boldsymbol{u}$ are, respectively, the stress and couple stress

* Received April 6, 1972.

1 Throughout this paper by Cosserat media we mean those with constrained rotation. 
tensors, and $s$ and $d$ denote the symmetric and deviatoric parts, respectively. On the other hand, the theorems established in [5] presuppose that

$$
\begin{aligned}
& 3 \lambda+2 \mu+\kappa \geq 0, \quad \mu \geq 0, \quad \kappa \geq 0, \quad \gamma \geq 0, \quad|\beta| \leq \gamma, \\
& 3 \alpha+\gamma+\beta \geq 0,
\end{aligned}
$$

where $\lambda, \mu, \kappa, \beta, \gamma$ are material constants such that the linear constitutive equations for the material are

$$
\begin{aligned}
\tau & =\lambda \nabla \cdot \mathbf{u I}+(\mu+\kappa) \nabla \mathbf{u}+\mu \mathrm{u} \nabla, \\
\mathbf{m} & =\alpha \nabla \cdot \phi \mathrm{I}+\beta \nabla \boldsymbol{\phi}+\gamma \boldsymbol{\phi} \nabla,
\end{aligned}
$$

in which $\mathbf{u}$ and $\phi$ are deformation and microrotation fields, respectively, and $\tau$ and $\mathbf{m}$ are the stress tensor and couple stress tensors, respectively.

The question as to whether or not the conditions (4) and (7) are necessary for uniqueness is of obvious theoretical interest. Therefore it is the purpose of the present work to show that, with limitation to the first boundary-value problem of elastodynamics of linear materials with microdeformations (surface deformations and microdeformations prescribed) and for bounded domains, the inequalities (4) and (5) can be relaxed without loss of uniqueness. Specifically, one finds that in these instances (4) and (5) may be replaced by the following less stringent assumptions:

$$
\begin{gathered}
\mu \geq 0, \quad \lambda+2 \mu \geq 0, \quad \eta \geq 0, \\
\lambda+2 \mu+\kappa \geq 0, \quad \mu+(\kappa / 2)>0, \quad \gamma \geq 0, \quad \alpha+\beta+\gamma \geq 0, \quad \kappa \geq 0 .
\end{gathered}
$$

2. A generalization of the uniqueness theorem in elastodynamics of Cosserat media. The displacement equations of motion of a linear, isotropic homogeneous Cosserat medium with constrained rotation take the form [4]

$$
\rho\left(\partial^{2} \mathbf{u} / \partial t^{2}\right)=\mu \nabla^{2} \mathbf{u}+(\lambda+\mu) \nabla \nabla \cdot \mathfrak{u}+\eta \nabla^{2}(\nabla \times \nabla \times \mathfrak{u})+\rho \mathbf{f}+\frac{1}{2} \nabla \times \rho \mathbf{c}
$$

in which $\mathbf{u}(x, t)$ is the displacement vector and $\mathbf{f}(x, t)$ and $\mathrm{c}(x, t)$ are, respectively, the body force and body couple per unit mass. Eq. (12), which must hold throughout the region of space $R$ occupied by the medium, is subject to the initial conditions

$$
\mathbf{u}(x, 0)=\overline{\mathbf{u}}(x), \quad \partial \mathbf{u} /\left.\partial t\right|_{(x, 0)}=\mathbf{v}(x) \text { in } R,
$$

where $\mathbf{u}(x)$ and $\mathbf{v}(x)$ are the prescribed initial distributions of displacement and velocity. In the first fundamental problem of elastodynamics of Cosserat media the accompanying boundary conditions are characterized by ${ }^{2}$

$$
\mathbf{u}(x, t)=\mathbf{U}(x, t), \quad \nabla \times \mathfrak{u}(x, t)=\mathbf{\Omega}(x, t) \text { on } s(0<t<\infty),
$$

where $s$ is the boundary of $R$ and $\mathrm{U}(x, t), \boldsymbol{\Omega}(x, t)$ are the given displacements and rotations on the surface, respectively.

Now let $\mathfrak{u}(x, t)$ and $\mathbf{u}^{*}(x, t)$ be two possible solutions of Eq. (12) subjected to conditions (13) and (14), and set $\mathfrak{u}^{*}-\mathfrak{u}=\mathbf{w}$. The governing equations for the determination of $w$ are

$$
\rho\left(\partial^{2} w / \partial t^{2}\right)=\mu \nabla^{2} w+(\lambda+\mu) \nabla \nabla \cdot w+\eta \nabla^{2}(\nabla \times \nabla \times w),
$$

s According to Koiter [6], conditions (14) make u kinematically admissible. 


$$
\begin{aligned}
& \mathbf{w}(x, 0)=\left.(\partial \mathrm{w} / \partial t)\right|_{(x, 0)}=0 \text { in } R, \\
& \mathbf{w}(x, t)=\nabla \times \mathbf{w}(x, t)=0 \text { on } s \quad(0<t<\infty) .
\end{aligned}
$$

Eq. (15) can be written alternatively as

$$
\begin{aligned}
\rho\left(\partial^{2} \mathrm{w} / \partial t^{2}\right)=(\lambda+2 \mu) \nabla \nabla \cdot \mathrm{w}-\mu \nabla \times \nabla \times \mathrm{w} & \\
& +\eta \nabla \times \nabla \times(\nabla \times \nabla \times \mathbf{w}) .
\end{aligned}
$$

Let $K(t)$ be the kinetic energy of the difference motion of the entire body, so that

$$
K(t)=\frac{\rho}{2} \int_{R}\left[\frac{\partial \mathrm{w}}{\partial t}\right]^{2} d R
$$

It may be shown with the help of certain vector identities and divergence theorem, selectively, that

$$
\begin{aligned}
\frac{d K}{d t}= & \int_{s}\left[(\lambda+2 \mu) \frac{\partial \mathrm{w}}{\partial t} \nabla \cdot \mathrm{w}+\mu \frac{\partial \mathrm{w}}{\partial t} \times \nabla \times \mathrm{w}+\eta(\nabla \times \nabla \times \nabla \times \mathrm{w}) \times \frac{\partial \mathrm{w}}{\partial t}\right. \\
& \left.+\eta\left(\frac{\partial}{\partial t}(\nabla \times \mathrm{w})\right) \times \nabla \times \nabla \times \mathrm{w}\right] \cdot d \mathrm{~s}-\frac{1}{2} \int_{R}\left[(\lambda+2 \mu) \frac{\partial}{\partial t}(\nabla \cdot \mathrm{w})^{2}\right. \\
& \left.+\mu \frac{\partial}{\partial t}(\nabla \times \mathrm{w})^{2}+\eta \frac{\partial}{\partial t}(\nabla \times \nabla \times \mathrm{w})^{2}\right] d R .
\end{aligned}
$$

It is now clear that with the aid of (18) and the second of (16) expression (19) can be integrated to yield

$$
\begin{aligned}
\frac{1}{2} \rho \int_{R}\left[\frac{\partial \mathrm{w}}{\partial t}\right]^{2} d R+ & \int_{R}\left[(\lambda+2 \mu)(\nabla \cdot \mathrm{w})^{2}\right. \\
& \left.+\mu(\nabla \times \mathrm{w})^{2}+\eta(\nabla \times \nabla \times \mathrm{w})^{2}\right] d R=K(t)+G(t)=c,
\end{aligned}
$$

in which $c$ is a constant of integration. With the aid of first of (16),

$$
c=0 \text {, }
$$

and therefore

$$
K(t)+G(t)=0 \quad(0 \leq t<\infty) .
$$

It is easily seen, with the aid of first of $(16)$, that for $\mathrm{w}(x, t)$ to be identically zero and thus ensure the uniqueness of solution $\mathfrak{u}(x, t)$, it is necessary and sufficient that:

$$
\lambda+2 \mu \geq 0, \quad \mu \geq 0, \quad \eta \geq 0 .
$$

These conditions are more relaxed than those obtained by Mindlin [4].

3. A generalization of the uniqueness theorem in elastodynamics of micropolar media. The governing equations of linear isotropic, homogeneous micropolar solid are

$$
\begin{aligned}
& \rho\left(\partial^{2} \mathfrak{u} / \partial t^{2}\right)=(\lambda+2 \mu+\kappa) \nabla \nabla \cdot \mathfrak{u}-(\mu+\kappa) \nabla \times \nabla \times \mathbf{u}+\kappa \nabla \times \boldsymbol{\phi}+\rho \mathbf{f}, \\
& \rho j\left(\partial^{2} \boldsymbol{\phi} / \partial t^{2}\right)=(\alpha+\beta+\gamma) \nabla \nabla \cdot \boldsymbol{\phi}-\gamma \nabla \times \nabla \times \boldsymbol{\phi}+\kappa \nabla \times \mathbf{u}-2 \kappa \boldsymbol{\phi}+\rho \mathbf{l},
\end{aligned}
$$


in which $\mathbf{u}(x, t), \boldsymbol{\phi}(x, t)$ are the macrodeformation vector and microrotation vector, respectively, $\mathbf{f}, 1$ are, respectively, the body force and body couple per unit mass, and $j$ is the microinertia. Eqs. (24) and (25), which must hold throughout the region of space $R$ occupied by the medium, are subject to the conditions

$$
\begin{aligned}
& \mathbf{u}(x, 0)=\overline{\mathbf{u}}(x), \quad \partial \mathbf{u} /\left.\partial t\right|_{(x, 0)}=\mathbf{v}(x) \quad \text { in } R, \\
& \boldsymbol{\phi}(x, 0)=\overline{\boldsymbol{\phi}}(x), \quad \partial \phi /\left.\partial t\right|_{(x, 0)}=\boldsymbol{\omega}(x) \text { in } R, \\
& \mathbf{u}(x, t)=\mathbf{U}(x, t) \text { on } s \text {, } \\
& \boldsymbol{\phi}(x, t)=\boldsymbol{\Phi}(x, t) \text { on } s .
\end{aligned}
$$

Now let $(\mathbf{u}, \boldsymbol{\phi})$ and $\left(\mathbf{u}^{*}, \boldsymbol{\phi}^{*}\right)$ be two possible states of equilibrium satisfying both (24) and (25) together with the conditions (26)-(29), and set $\mathbf{w}=\mathbf{u}^{*}-\mathfrak{u}, \boldsymbol{v}=\boldsymbol{\phi}^{*}-\boldsymbol{\phi}$. The governing equations for the determination of $w$ and $v$ are

$$
\begin{aligned}
\rho\left(\partial^{2} \mathbf{w} / \partial t^{2}\right) & =(\lambda+2 \mu+\kappa) \nabla \nabla \cdot \mathbf{w}-(\mu+\kappa) \nabla \times \nabla \times \mathbf{w}+\kappa \nabla \times v, \\
\rho j\left(\partial^{2} \mathbf{v} / \partial t^{2}\right) & =(\alpha+\beta+\gamma) \nabla \nabla \cdot v-\gamma \nabla \times \nabla \times v+\kappa \nabla \times \mathbf{w}-2 \kappa v, \\
\mathbf{w}(x, 0) & =\left.(\partial \mathbf{w} / \partial t)\right|_{(x, 0)}=\boldsymbol{v}(x, 0)=\left.(\partial v / \partial t)\right|_{(x, 0)}=0 \text { in } R, \\
\mathbf{w}(x, t) & =v(x, t)=0 \text { on } s .
\end{aligned}
$$

Let $T=T_{1}+T_{2}$, where

$$
T_{1}=\frac{\rho}{2} \int\left[\frac{\partial \mathrm{w}}{\partial t}\right]^{2} d R, \quad T_{2}=\frac{\rho j}{2} \int_{R}\left[\frac{\partial v}{\partial t}\right]^{2} d R,
$$

It may be shown with the help of certain vector identities and divergence theorems that

$$
(d T / d t)+(d G / d t)=0, \quad 0<t<\infty,
$$

where

$$
\begin{aligned}
G=\frac{1}{2} \int_{R}\left[(\lambda+2 \mu+\kappa)(\nabla \cdot w)^{2}\right. & +[\mu+(\kappa / 2)](\nabla \times w)^{2}+\gamma(\nabla \times v)^{2} \\
& \left.+(\alpha+\beta+\gamma)(\nabla \cdot v)^{2}+2 \kappa\left(v-\frac{1}{2} \nabla \times w\right)^{2}\right] d R .
\end{aligned}
$$

From (35),

$$
T(t)+G(t)=c \quad(0<t<\infty) .
$$

But noting that $T(0)=G(0)=0$ yields $c=0$; thus

$$
T(t)+G(t)=0, \quad(0 \leq t<\infty) .
$$

It is easily seen, with the aid of (26) and (27), that for $\mathrm{w}(x, t), \mathrm{v}(x, t)$ to be identically zero and thus ensuring the uniqueness of solution of $\mathbf{u}(x, t) \boldsymbol{\phi}(x, t)$, it is necessary and sufficient that

$$
\lambda+2 \mu+\kappa \geq 0, \quad \mu+\kappa / 2 \geq 0, \quad \gamma \geq 0 \quad \kappa \geq 0 \quad \alpha+\beta+\gamma \geq 0 .
$$

These conditions are more relaxed than those established by Eringen [5]. 


\section{REFERENCES}

[1] G. Kirchhoff, J. reine und angew. Math. 56, 285 (1859)

[2] F. Neumann, Vorlesungen uber die Theorie der Elasticitat der festen Korper und des Lichtathers, B. G. Teubner, Leipzig, 1885

[3] M. E. Gurtin and Eli Sternberg, Quart. Appl. Math., 29, 169 (1961)

[4] R. D. Mindlin and H. F. Tiersten., Arch. Rat. Mech. Anal., 5, 415 (1962)

[5] A. C. Eringen, J. Math. Mech. 15, 6 (1966)

[6] W. T. Koiter, Proc. Kon. Nederl. Akad. Wetenschappen B67, 17-44 (1964) 